\title{
"Textural analysis of multiparametric MRI detects transition zone prostate cancer"
}

\author{
Harbir S. Sidhu ${ }^{1,2} \cdot$ Salvatore Benigno $^{1} \cdot$ Balaji Ganeshan $^{3} \cdot$ Nikos Dikaios $^{1} \cdot$ \\ Edward W. Johnston 1,2 - Clare Allen ${ }^{2}$ - Alex Kirkham ${ }^{2}$ - Ashley M. Groves ${ }^{2,3}$. \\ Hashim U. Ahmed ${ }^{2,4}$ • Mark Emberton ${ }^{2,4}$ • Stuart A. Taylor ${ }^{1,2}$ - Steve Halligan ${ }^{1,2}$. \\ Shonit Punwani ${ }^{1,2,5}$
}

Received: 7 June 2016 /Revised: 10 August 2016 / Accepted: 22 August 2016 / Published online: 12 September 2016

(C) The Author(s) 2016. This article is published with open access at Springerlink.com

\begin{abstract}
Objectives To evaluate multiparametric-MRI (mpMRI) derived histogram textural-analysis parameters for detection of transition zone (TZ) prostatic tumour.

Methods Sixty-seven consecutive men with suspected prostate cancer underwent $1.5 \mathrm{~T}$ mpMRI prior to template-mappingbiopsy (TPM). Twenty-six men had 'significant' TZ tumour. Two radiologists in consensus matched TPM to the single axial slice best depicting tumour, or largest TZ diameter for those with benign histology, to define single-slice whole TZ-regions-ofinterest (ROIs). Textural-parameter differences between singleslice whole TZ-ROI containing significant tumour versus benign/insignificant tumour were analysed using Mann Whitney U test. Diagnostic accuracy was assessed by receiver operating characteristic area under curve (ROC-AUC) analysis cross-validated with leave-one-out (LOO) analysis.

Results ADC kurtosis was significantly lower $(\mathrm{p}<0.001)$ in $\mathrm{TZ}$ containing significant tumour with ROC-AUC 0.80
\end{abstract}

Shonit Punwani

shonit.punwani@gmail.com

1 Centre for Medical Imaging, University College London, 3rd Floor East, 250 Euston Road, London NW1 2BU, UK

2 University College London Hospitals NHS Foundation Trust, 235 Euston Road, London NW1 2BU, UK

3 Institute of Nuclear Medicine, University College London, University College Hospital, 235 Euston Road, London NW1 2BU, UK

4 Research Department of Urology, University College London, 3rd Floor, Charles Bell House 67 Riding House Street, London W1P $7 \mathrm{NN}, \mathrm{UK}$

5 Centre for Medical Imaging, University College London and University College London Hospitals NIHR Biomedical Research Centre, 250 Euston Road, London NW1 2BU, UK
(LOO-AUC 0.78); the difference became non-significant following exclusion of significant tumour from single-slice whole TZ-ROI $(p=0.23)$. T1-entropy was significantly lower $(\mathrm{p}=0.004)$ in TZ containing significant tumour with ROCAUC 0.70 (LOO-AUC 0.66) and was unaffected by excluding significant tumour from TZ-ROI $(\mathrm{p}=0.004)$. Combining these parameters yielded ROC-AUC 0.86 (LOO-AUC 0.83). Conclusion Textural features of the whole prostate TZ can discriminate significant prostatic cancer through reduced kurtosis of the ADC-histogram where significant tumour is included in TZ-ROI and reduced T1 entropy independent of tumour inclusion.

Key Points

- MR textural features of prostate transition zone may discriminate significant prostatic cancer.

- Transition zone (TZ) containing significant tumour demonstrates a less peaked ADC histogram.

- TZ containing significant tumour reveals higher postcontrast T1-weighted homogeneity.

- The utility of MR texture analysis in prostate cancer merits further investigation.

Keywords Magnetic Resonance Imaging $\cdot$ Prostate $\cdot$ Cancer . Diagnosis $\cdot$ Image Processing

$\begin{array}{ll}\text { Abbreviations } \\ \text { MCCL } & \text { Maximum cancer core length }(\mathrm{mm}) \\ \text { mpMRI } & \text { Multi-parametric MRI } \\ \text { MRTA } & \text { MRI textural analysis } \\ \text { TPM } & \begin{array}{l}\text { Transperineal template prostate mapping } \\ \text { biopsy }\end{array} \\ \text { TZ } & \text { Transition zone of the prostate }\end{array}$

Abbreviations

MCCL Maximum cancer core length (mm)

mpMRI Multi-parametric MR

MRTA MRI textural analysis

TZ Transition zone of the prostate 


\section{Introduction}

Management of early prostate cancer has been revolutionised by the use of multi-parametric MRI (mpMRI; using T2 and T1 weighted, diffusion-weighted and contrast- enhanced imaging) [1]. Nonetheless, transition zone (TZ) tumours remain more difficult to appreciate on mpMRI studies [2]; with reported sensitivity/specificity for detection of $0.53 / 0.83 \mathrm{com}-$ pared with $0.80 / 0.97$, respectively, for peripheral zone (PZ) tumours [1]. TZ tumour signal homogeneity has been advocated as a discriminator of significant grade disease at mpMRI and has been incorporated into the recently revised 'Pi-RADS 2' guidelines [3]; endorsed by the European Society of Urogenital Radiology and the American College of Radiology.

Textural analysis is an image-processing technique that can assess image signal heterogeneity (both at and beyond that appreciated by the human eye) by quantifying the coarseness and regularity of the spatial distribution of pixel grey level values within normal and pathological tissue. Macroscopic heterogeneity in medical images may reflect microscopic heterogeneity at the histopathological level, particularly in oncological imaging with recent demonstrations of utility in tumour detection/grading, prognosis and treatment response $[4,5]$. Compared to CT, MRI offers the advantages of improved soft tissue contrast resolution and of a wealth of imaging data afforded by a multi-parametric approach. Recent studies have used MR textural analysis (MRTA) for lesion detection, classification, treatment response-evaluation and prediction for example in breast, brain, and rectal cancer [6-8]. A number of approaches to texture analysis exist, with one approach being quantification of features through histogram analysis [9].

It is possible the additional tissue heterogeneity data provided by textural analysis could augment the diagnostic accuracy of radiologists in detecting TZ tumours, where lesions tend to be subtle and difficult to differentiate from adjacent benign nodular tissue. In this study, image analysis was performed on a whole TZ basis with the aim of obviating the need for radiological pre-identification of tumour, thus increasing potential utility in computer-aided diagnosis (CAD) and to minimise inherent difficulties of small lesion contouring. The purpose of this study was to evaluate multiparametric MRI (mpMRI) derived histogram textural analysis parameters [9] for detection of transition zone (TZ) prostatic tumour.

\section{Materials and methods}

Our local institutional review board approved the study and waived the requirement for individual consent for this retrospective study of consecutive patient data acquired as part of routine clinical care (R\&D No: 12/0195).

\section{Patient cohort}

The cohort comprised men with clinically suspected prostate cancer undergoing prostatic mpMRI prior to ' 20 zone' template prostate mapping (TPM) biopsies (within 12 months) between 1 January 2010 to 31 December $2012(n=210)$. Men who had a peripheral zone tumour, undergone biopsy within 6 months prior to mpMRI, received previous treatment for prostate cancer, had metallic hip prostheses, or had incomplete mpMRI and/or TPM data sets were excluded $(n=143)$. In total sixty-seven men with mean age 63.4 years $(45$ 80 years), mean PSA $9.2 \mathrm{ng} / \mathrm{ml}(0.2-39.0 \mathrm{ng} / \mathrm{ml})$ and mean gland volume of $42.9 \mathrm{ml}(15-101 \mathrm{ml})$ were accrued. Of these 29/67 (43\%) had no cancer, 26/67 (39\%) had 'significant' TZ cancer and 12/67 (18\%) had 'insignificant' TZ cancer (see below). Table 1 summarizes cohort demographics.

\section{Multi-parametric magnetic resonance imaging}

Subjects underwent 1.5T magnet mpMRI (Avanto, Siemens, Erlangen, Germany) with pelvic-phased array coil, following intravenous spasmolytic (Buscopan; Boehringer Ingelheim, Germany) $0.2 \mathrm{mg} / \mathrm{kg}$ (maximum $20 \mathrm{mg}$ ) to minimize bowel peristalsis. Full mpMRI parameters are given in Table 2. Figure 1 shows an example mpMRI with a significant TZ tumour.

\section{Transperineal template-prostate-mapping biopsy}

TPM followed mpMRI with a median interval of 56 days ( 2 to 214 days); method as previously described [10, 11]. In brief, systematic biopsy of the whole gland was performed through a brachytherapy template-grid and 5-mm sampling frame, giving a uniform sampling density of approximately 1 core/cc of prostatic tissue. Biopsies were grouped and potted into 20 zones, modified from the technique reported by Barzell et al. [12].

\section{Histopathology review}

Subjects were grouped according to a previously used definition of cancer significance [13] whereby TPM maximum cancer core length (MCCL) values were used to infer volume, following demonstration that a TPM MCCL of $\geq 4 \mathrm{~mm}$ approximates to a tumour volume of $\geq 0.2 \mathrm{ml}$ [14].

Clinically significant disease was defined as $\geq$ Gleason $3+$ $4 \mathrm{OR} \geq 4 \mathrm{~mm}$ MCCL, while other disease (i.e., 'low risk' $<4 \mathrm{~mm}$ MCCL $\mathrm{AND} \leq$ Gleason $3+3$ ) was classified as nonsignificant and grouped with patients demonstrating benign TZ histology [15]. 
Table 1 Summary of demographic and ROI areas for recruited patients categorised by benign/insignificant transition (TZ) pathology and significant $\mathrm{TZ}$ tumours $(\mathrm{MCCL}=$ maximum cancer core length,
$\mathrm{G}=$ Gleason grade, $\mathrm{PSA}=$ prostate specific antigen serum concentration, $\mathrm{TPM}=$ template mapping biopsy)

\begin{tabular}{|c|c|c|c|c|c|c|c|c|}
\hline Cancer significance & Number $(\%)$ & $\begin{array}{l}\text { Mean Age } \\
\text { years } \\
(\text { range) }\end{array}$ & $\begin{array}{l}\text { Mean PSA } \\
\text { ng/dL } \\
\text { (range) }\end{array}$ & $\begin{array}{l}\text { Mean prostate } \\
\text { volume } \mathrm{ml} \\
\text { (range) }\end{array}$ & $\begin{array}{l}\text { Median time } \\
\text { interval mpMRI } \\
\text { to TPM days } \\
\text { (range) }\end{array}$ & $\begin{array}{l}\text { Mean area TZ } \\
\text { ROI cm }{ }^{2} \\
\text { (Total; range) }\end{array}$ & $\begin{array}{l}\text { Mean area } \\
\mathrm{TZ} \text { tumour } \mathrm{cm}^{2} \\
\text { (Total; range) }\end{array}$ & $\begin{array}{l}\text { Percentage } \mathrm{TZ} \\
\text { tumour area/TZ } \\
\text { area (Total; range) }\end{array}$ \\
\hline $\begin{array}{l}\text { Benign OR } \\
\text { Insignificant } \\
\quad(<4 \mathrm{~mm} \text { MCCL } \\
\quad \mathrm{AND} \leq \mathrm{G} 3+3)\end{array}$ & $\begin{array}{l}41 \\
(61 \%)\end{array}$ & $\begin{array}{l}64 \\
64(45-79)\end{array}$ & $\begin{array}{l}8.6 \\
(0.2-39)\end{array}$ & $\begin{array}{l}47.0 \\
(20-101)\end{array}$ & $\begin{array}{l}54 \\
(6-214)\end{array}$ & $\begin{array}{l}40.0 \\
(n=41 ; \\
10.0-68.9)\end{array}$ & $\begin{array}{l}0.76 \\
(n=12 \\
0.52-1.22)\end{array}$ & $\begin{array}{l}2.4 \\
(n=12 ; \\
1.1-3.7)\end{array}$ \\
\hline $\begin{array}{l}\text { Significant } \\
\qquad(\geq 4 \mathrm{~mm} \mathrm{MCCL} \\
\quad \mathrm{OR} \geq \mathrm{G} 3+4)\end{array}$ & $\begin{array}{l}26 \\
(39 \%)\end{array}$ & $\begin{array}{l}63 \\
(52-80)\end{array}$ & $\begin{array}{l}9.9 \\
(0.3-35)\end{array}$ & $\begin{array}{l}37.2 \\
(15-78)\end{array}$ & $\begin{array}{l}56 \\
(16-145)\end{array}$ & $\begin{array}{l}29.9 \\
(n=26 ; \\
9.3-61.2)\end{array}$ & $\begin{array}{l}1.45 \\
(n=26 ; \\
0.65-3.80)\end{array}$ & $\begin{array}{l}5.3 \\
(n=26 ; \\
1.7-14.0)\end{array}$ \\
\hline
\end{tabular}

\section{Histology-MRI Matching}

Two radiologists in consensus (HSS, SP; with 8 years and 4 years of experience, respectively, for mpMRI interpretation), aware of histopathological findings, reviewed each dataset using Osirix (version 3.5.1; Geneva, Switzerland) and matched the single axial slice depicting the TZ focus most suspicious for disease to location of disease confirmed according to histopathology. If no tumour was present, the radiologists selected the slice with the largest $\mathrm{TZ}$ anteroposterior diameter. Where biopsy-positive significant tumour was present, the radiologists also contoured significant tumours on each ADC, T2 and T1 weighted TZ single slice image to analyse the effect of initially including and then excluding significant tumours from the single-slice whole TZ ROI drawn by a third radiologist (below). TZ tumour contoured on T2 images was used for tumour area estimation (relative to total TZ area).

A third radiologist (SB; with 2 years of experience for mpMRI interpretation), blinded to biopsy data and earlier tumour region of interest (ROI) placement, contoured solely the entire $\mathrm{TZ}$ on the selected slice, on matched ADC, T2 and early post-contrast T1 weighted images for each patient for subsequent MRTA.

The percentage of $\mathrm{TZ}$ replaced by tumour was quantified by [(tumour area / TZ area)*100].
Table 2 Multi-parametric MRI sequence parameters used for study; *dynamic contrast enhanced MRI$0.2 \mathrm{ml} / \mathrm{kg}$ intravenous gadolinium contrast agent injected at the beginning of acquisition 6 at $3 \mathrm{ml} / \mathrm{s}$ followed by a saline flush of $20 \mathrm{ml}$; T2w TSE - T2 weighted turbo spin echo; EPI-DWI - echo planar imaging - diffusion weighted imaging; FLASH - fast low angle shot. Note coronal T2 acquisition was not used for image analysis (though remains part of the clinical scan). Apparent diffusion coefficient (ADC) map automatically generated from the four b-values (mono-exponential). 'Early post contrast $\mathrm{T} 1$ ' image refers to the second image temporally from the point at which contrast first appears in the prostate gland (imaging every 16 seconds)

\begin{tabular}{lccc}
\hline & $\begin{array}{c}\text { T2w TSE } \\
\text { axial/coronal }\end{array}$ & EPI DWI & T1 3D FLASH* \\
\hline Repetition time (ms) & $5170 / 5240$ & 2100 & 5.61 \\
Echo time (ms) & $92 / 104$ & 98 & 2.5 \\
Flip angle (degrees) & $180 / 150$ & 90 & 15 \\
Echo train length & $22 / 24$ & 172 & $\mathrm{n} / \mathrm{a}$ \\
Bandwidth (Hz/Px) & $190 / 190$ & 968 & 300 \\
Field of view (mm) & $180 / 180$ & 260 & 260 \\
Phase FoV \% & $100 / 100$ & 100 & 100 \\
Slice thickness (mm) & $3 / 3$ & 5 & 3 \\
Slice gap (mm) & $0.3 / 0.3$ & 0 & 0.6 \\
Averages & $2 / 2$ & 16 & 1 \\
Phase encoding direction & $\mathrm{A}>\mathrm{P} / \mathrm{R}>\mathrm{L}$ & $\mathrm{A}>\mathrm{p}$ & $\mathrm{A}>\mathrm{P}$ \\
Fat saturation & No / No & Yes & Yes \\
Base matrix & $256 / 256$ & 172 & 192 \\
Matrix phase \% & $95 / 95$ & 100 & 100 \\
b-values (s.mm ${ }^{-2}$ ) & $\mathrm{n} / \mathrm{a}$ & $0,300,500,1000$ & $\mathrm{n} / \mathrm{a}$ \\
Number of acquisitions & $1 / 1$ & $10 \mathrm{~m}$ \\
Temporal resolution (s) & $\mathrm{n} / \mathrm{a}$ & $3 \mathrm{~m} 39 \mathrm{~s}$ & 16 \\
Total acquisition time (min) & $3 \mathrm{~m} 54 \mathrm{~s} / 4 \mathrm{~m} 18 \mathrm{~s}$ & & \\
\hline
\end{tabular}


Fig. 1 Demonstrates single slice axial images of significant tumours (arrow): (a) T2 weighted; (b) ADC map; (c) pre-contrast T1 weighted; and (d) early post contrast $\mathrm{T} 1$ weighted images in a 74-year-old patient with an anterior right transition zone tumour (Gleason $3+4$; maximum cancer core length $11 \mathrm{~mm}$ ) prior to transition zone contouring and histogram analysis
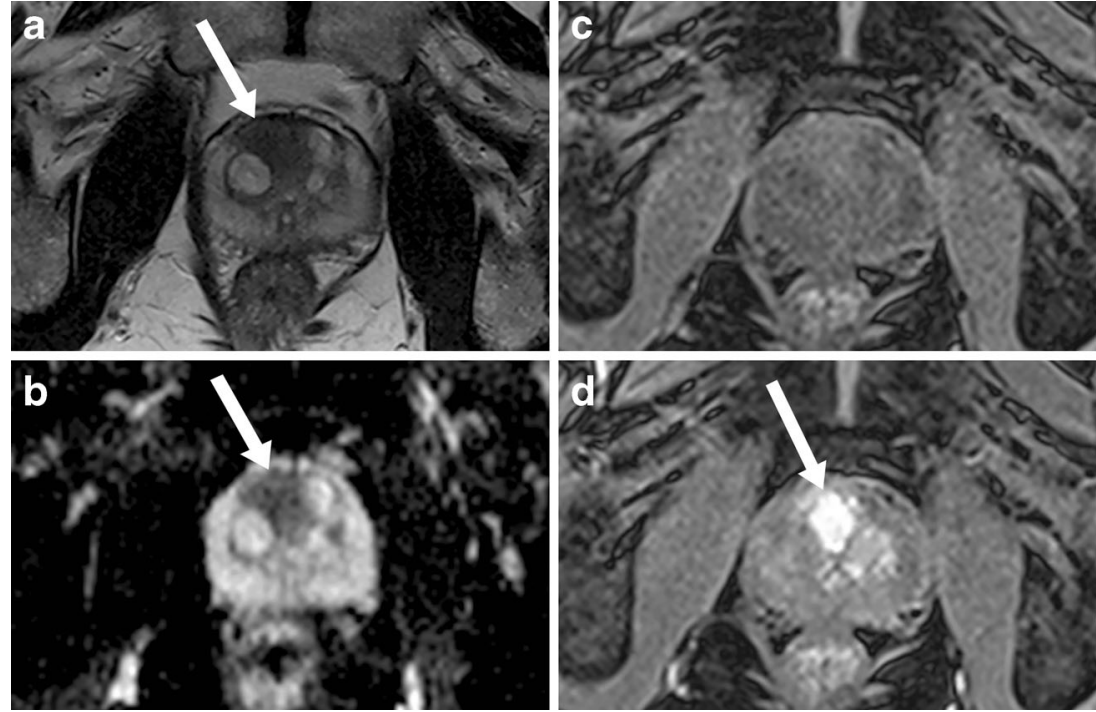

\section{MR Textural Analysis (MRTA)}

The ADC map, T2 and early post-contrast $\mathrm{T} 1$ weighted segmented $\mathrm{TZ}$ regions from the selected slice (containing and initially including tumour where present) underwent MRTA (BG, with 9 years of experience in texture analysis) using proprietary TexRAD research software (version 3.3, TexRAD Ltd, Feedback Plc, Cambridge, UK) with automated texture parameter extraction. A subsequent separate analysis examined single-slice whole TZ ROIs excluding significant tumours to assess the effect on textural parameters.

MRTA comprised image histogram analysis to quantify first-order statistics of entropy, skewness, and kurtosis of the TZ ROIs. These parameters reflect, to varying degrees, the number, intensity and variability of areas of high and low signal intensity within the TZ [9]. Absolute T2 and T1 weighted signal intensities are not comparable across patients without standardisation, and; therefore, T1 and T2 mean pixel, mean positive pixel and standard deviation values were not analysed further (unlike mean ADC). By contrast, measurement of entropy, kurtosis and skewness rely on the shape of the histogram, i.e., relationships between pixel intensities and not on the absolute pixel intensity values.

\section{Statistical Analysis}

For each individual textural variable, the significance of difference between non-significant/benign $\mathrm{TZ}$ and significant tumours containing $\mathrm{TZ}$ were assessed using the two tailed Mann Whitney $\mathrm{U}$ test (statistical significance assigned at $p<0.05$ ). These analyses were repeated after exclusion of significant tumour area from the single-slice whole TZ ROI to determine if the observed differences in textural parameters could be directly attributed to inclusion of significant tumour within the analyzed TZ ROI. Receiver-operating characteristics
(ROC) analyses characterised the performance of TZ textural features extracted from each of ADC, T1 and T2 weighted TZ $\mathrm{ROI}$ to predict significant $\mathrm{TZ}$ prostate cancer.

The area under the ROC curve (ROC AUC) and $95 \%$ confidence intervals for each parameter identified the best performing individual parameters and in combination using multivariate ROC-AUC analysis [16]. Leave-one-out (LOO) analysis [17] validated classification performance for best performing univariate and combined parameters.

All statistical analyses were performed using SPSS statistics for Windows (version 16; IBM, Armonk, NY) and MedCalc for Windows (version 9.2.0.0; MedCalc software, Mariakerke, Belgium).

\section{Results}

\section{Patient cohort}

Mean tumour area was $1.45 \mathrm{~cm}^{2}$ ( 0.65 to 3.8 ) for patients with significant tumour at histology. Mean TZ area was $42.4 \mathrm{~cm}^{2}$ (10.0 to 68.9) for patients with benign histology; $33.9 \mathrm{~cm}^{2}$ (18.7 to 58.8) for patients with non-significant cancer, and $29.9 \mathrm{~cm}^{2}$ (9.3 to 61.2) for patients with significant cancer. Mean proportion of $\mathrm{TZ}$ replaced by tumour was $5.3 \%$ (range 1.7 to $14.0 \%$ ) for patients with significant tumour (Table 1). Table 3 denotes the median values and interquartile ranges of whole TZ MR textural parameters for patients with non-significant/benign and for patients with significant tumour both including and excluding the significant tumour. P-values denote the statistical significance of differences between whole $\mathrm{TZ}$ for patients with non-significant/benign histology and patients with significant tumour for TZ ROIs (again including and excluding the significant tumour). The ROC-AUC for whole TZ textural parameters for each of the three MRI 


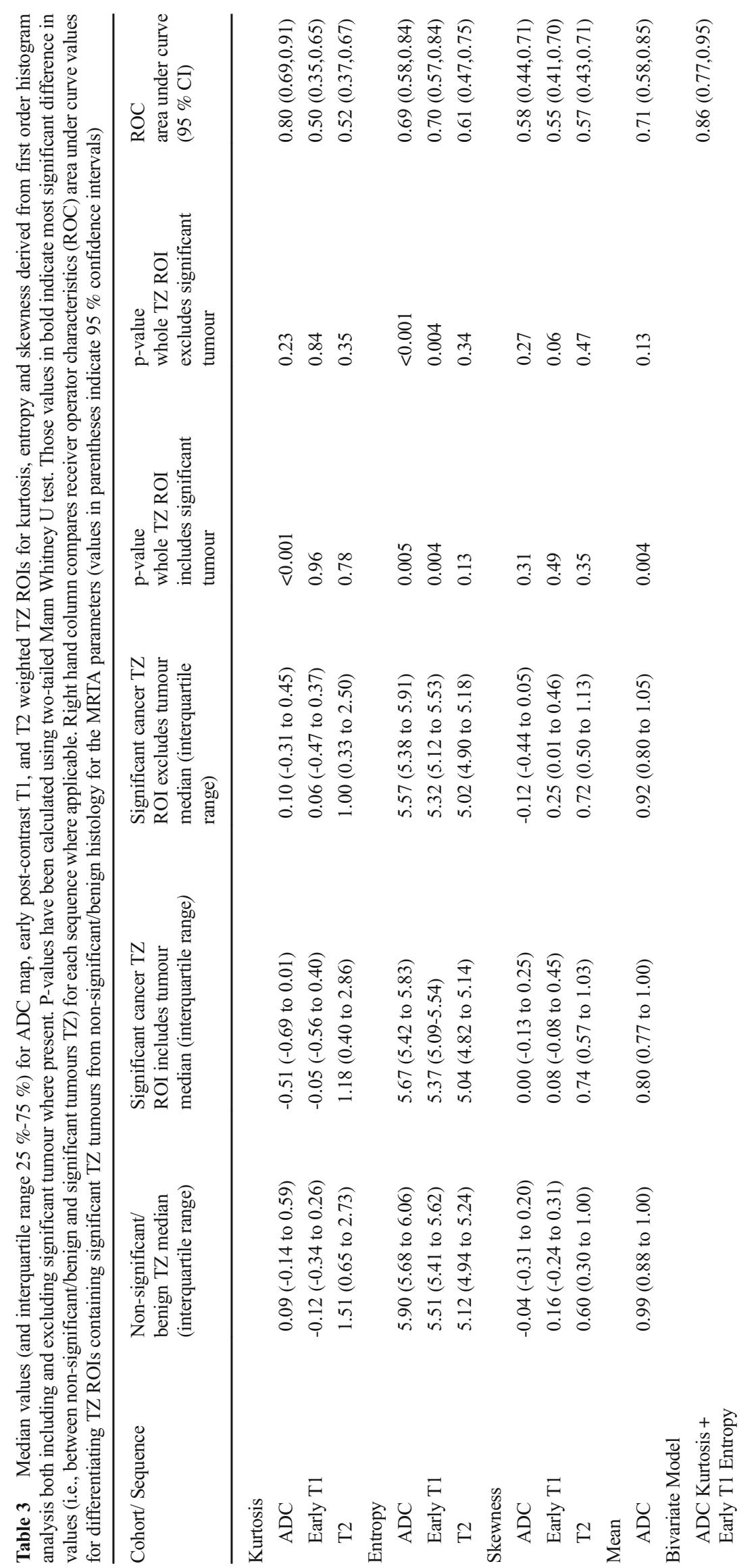


sequences, for classification of a $\mathrm{TZ}$ image as containing significant tumour, are also detailed.

\section{TZ textural metrics}

All analyses were performed on a whole TZ basis (initially incorporating significant tumour where present). Mean and median TZ ADC were significantly higher $(0.99$ and $0.97 \mathrm{x}$ $10-3 \mathrm{~mm} 2 / \mathrm{s}$, respectively, range 0.67 to 1.77 ) for patients with non-significant/benign histology than those with significant tumour $(0.79$ and $0.77 \times 10-3 \mathrm{~mm} 2 / \mathrm{s}$ respectively, range 0.07 to 1.22$)(p=0.004)$.

\section{Kurtosis}

Median ADC, T1 and T2 kurtosis were 0.09, -0.12 and 1.51, respectively, for non-significant/benign histology versus $0.51,-0.05$ and 1.18 , respectively, for TZ containing significant tumour. Median ADC kurtosis was significantly lower for patients with significant tumour $(p<0.001)$; whereas median T1 and T2 kurtosis were not significantly different between the groups ( $p=0.96$ and 0.78 , respectively).

\section{Entropy}

Median ADC, T1 and T2 entropy were 5.90, 5.51 and 5.12, respectively, for non-significant/benign histology and 5.67, 5.37 and 5.04, respectively, for TZ containing significant tumour. Median ADC and T1 entropy were lower for patients with significant tumour ( $p=0.005$ and 0.004 , respectively) whilst T2 entropy did not reach significance $(p=0.13)$.

\section{Skewness}

Median ADC, T1 and T2 skewness were -0.04, 0.16 and 0.60 , respectively, for non-significant/benign histology versus 0.00 , 0.08 and 0.74 , respectively, for TZ containing significant tumours. There were no significant differences between the two groups ( $p=0.31$ to 0.49$)$.

\section{Effect of excluding significant tumours on whole TZ textural metrics}

Following exclusion of significant tumours from the singleslice whole TZ ROI, there was no longer a significant difference between median ADC value $(p=0.13)$, when patients with those significant tumours were compared with those with non-significant/benign histology.

\section{Kurtosis}

Following exclusion of significant tumours from the singleslice whole TZ ROI, there was no longer a significant difference between median ADC kurtosis, when patients with significant tumours were compared with those with non-significant/benign histology $(p=0.23)$. Median T1 and T2 kurtosis differences remained non-significant ( $p=0.84$ and 0.35 , respectively; Table 3).

\section{Entropy}

Significant differences between T1 and ADC entropy remained, when patients with significant tumours and those with non-significant/benign histology were compared following exclusion of significant tumours from the former ROI ( $p$ $<0.01$, Table 3).

\section{Skewness}

Median ADC, T1 and T2 skewness did not demonstrate any consistent difference between patients with significant tumours and those with non-significant/benign histology following exclusion of significant tumours from the TZ ROI.

Box-and-whiskers plot of the best performing textural parameters are illustrated in Fig. 2.

\section{Disease classification by univariate textural metrics}

The best performing classifier for $\mathrm{ADC}$ was kurtosis $(0.80$; $95 \%$ CI 0.69 to 0.91 ). Entropy yielded the highest AUC for early $\mathrm{T} 1$ post-contrast image $(0.70 ; 95 \%$ CI 0.57 to 0.84$)$. This analysis did not show significant differences on $\mathrm{T} 2$ weighted whole $\mathrm{TZ}$ textural analysis with the best performing T2 parameter being T2 entropy (AUC of $0.61 ; 95 \% \mathrm{CI} 0.47$ to 0.75). LOO validation demonstrated ROC-AUC 0.78 (95\% CI 0.66 to 0.90$)$ for ADC kurtosis and 0.66 (95\% CI 0.52 to 0.80 ) for $\mathrm{T} 1$ entropy.

\section{Disease classification by multivariate textural metrics}

ROC-AUC for the best performing significant univariate parameters and bivariate combination of these parameters are shown in Fig. 3. The two best performing parameters for ADC (kurtosis) and T1 (entropy) combined in the bivariate model gave ROC-AUC of 0.86 (95\% CI 0.77 to 0.95 ). LOO analysis of this bivariate model yielded ROC-AUC 0.83 (95\% CI 0.74 to 0.93 ) and is shown in Fig. 4.

\section{Discussion}

This study evaluated the diagnostic accuracy of textural parameters, derived from clinical prostate mpMRI, for detection of TZ cancer. Previous work has confirmed that quantitative mpMRI parameters (e.g., ADC) can differ between benign and cancerous TZ regions [18]. Unlike previous studies, here 
Fig. 2 Box plots showing best performing textural discriminators of TZ ROIs containing significance and nonsignificance using ADC kurtosis and early post-contrast $\mathrm{T} 1$. In each box plot the box indicates interquartile range; line indicates median and whiskers indicate most deviated data points/range. Two tailed Mann Whitney U pvalues are also given for each parameter

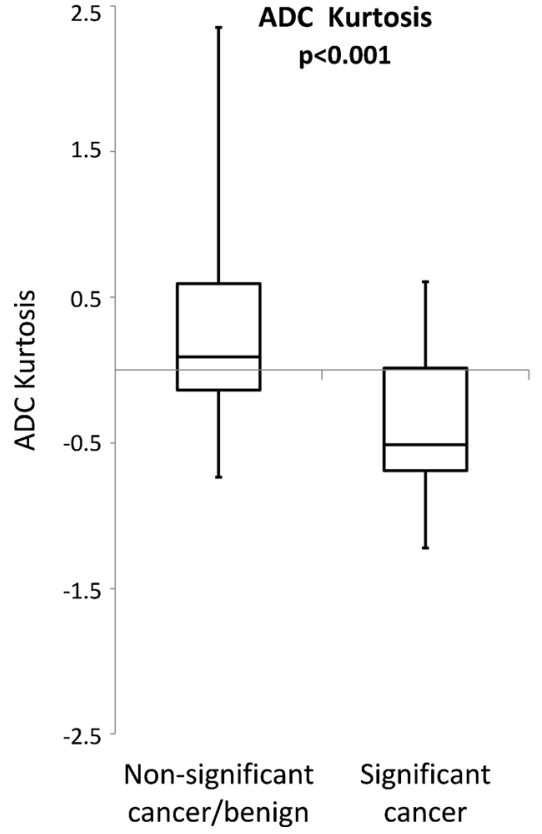

we derived single-slice whole TZ textural parameters (including cancer pixels where cancer was present) and evaluated differences in the histographic pixel distribution between patients with and without significant cancer.

We found that textural features from an image of the entire TZ are altered significantly, when containing even a small proportion of significant cancer, which no longer holds true (for best performing textural parameter) when the same tumour is excluded from the analysis. Overall, classification of TZ tumour-containing slices by best performing single textural parameter and/or bivariate combination (ROC-AUC 0.80 to $0.86)$ was comparable with previously reported visual detection of TZ tumour by radiologists (ROC-AUC 0.73 to 0.84) $[19,20]$.

Kurtosis is a measure of histogram "peakedness". Positive kurtosis indicates a more peaked distribution of pixel signal intensities. We found reduced ADC kurtosis was the best univariate classifying textural feature (ROC-AUC 0.80 on ADC images) on a whole $\mathrm{TZ}$ basis and demonstrated a higher ROCAUC than the non-textural parameter of ADC mean (ROCAUC 0.71 on ADC images). A larger cohort is required to test the statistical significance of this difference. We expect textural measures, based on the relationship of pixels in a given ROI rather than absolute pixel intensities, are likely more robust to
Fig. 3 Receiver operating characteristic (ROC) curves of the two best performing textural features and bivariate combination for discrimination of transition zone ROIs containing significant prostatic tumours from non-significant $\mathrm{TZ}$ with area under curve (AUC) values as shown

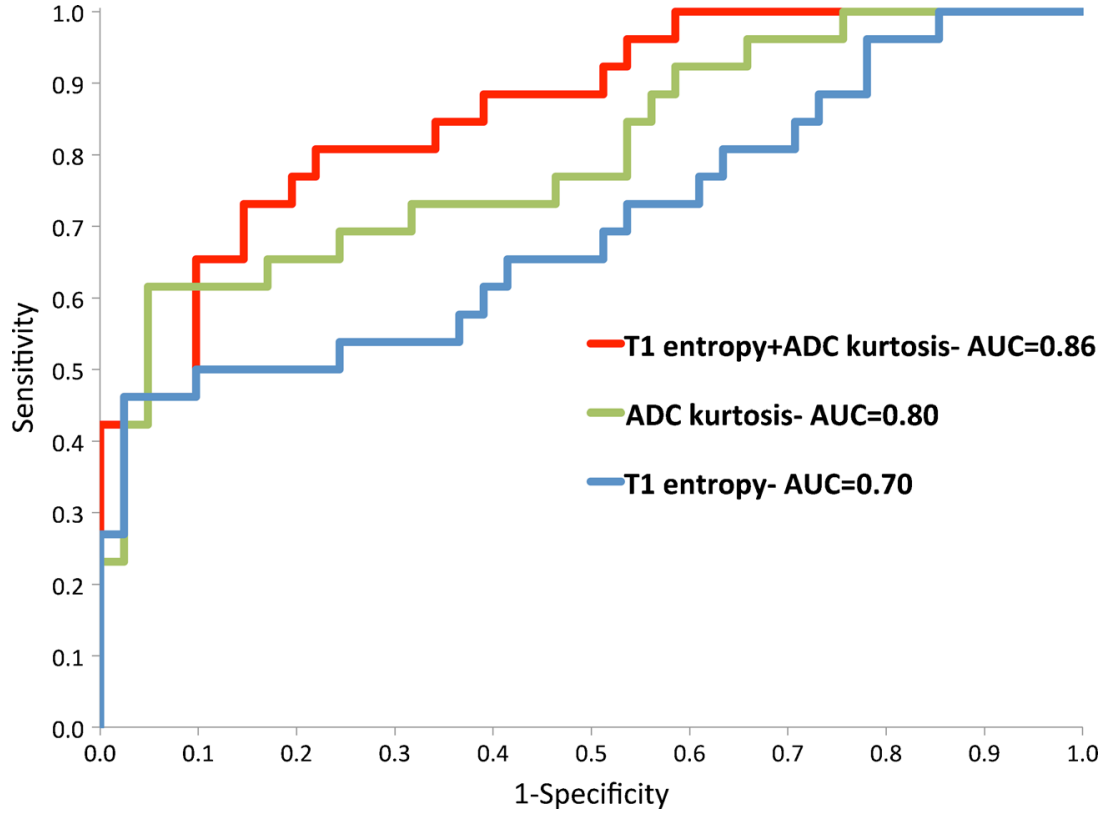


Fig. 4 Receiver operating characteristic (ROC) curves of the two best performing textural features and bivariate combination for discrimination of TZ ROIs containing significant prostatic tumours from nonsignificant TZ ROIs after leaveone-out (LOO) analysis with area under curve (AUC) values as shown

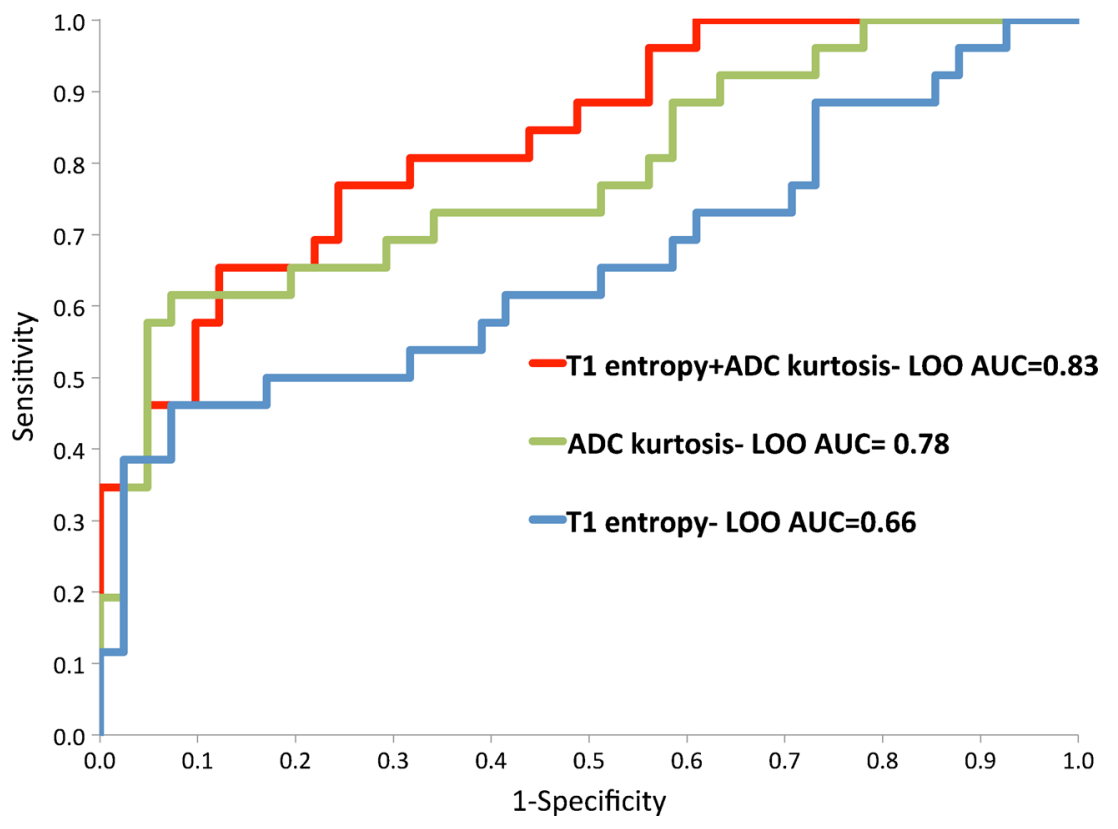

variations between individuals and scanners. Whilst kurtosis measures from $\mathrm{T} 2$ and early post-contrast $\mathrm{T} 1$ weighted images performed less well for the detection of cancer. In spite of the relatively modest ratio of significant tumour compared to remaining TZ area ( $\sim 5 \%)$, we have found that exclusion of this radiologically visible tumour from the single-slice whole TZ ROI results in loss of significance in the difference in ADC kurtosis between the two groups. We posit this to be the result of elimination of a second differing signal intensity 'population' (i.e., a low ADC significant tumour) from the TZ ROI resulting in normalisation of kurtosis when compared to the non-significant/benign cohort reflected in more 'peaked' values. Wibner et al. [21] have previously demonstrated the utility of ADC-derived textural parameters in TZ tumour detection and differentiating grade based on lesion analysis. The textural analysis approach may obviate previously described efforts to normalise by using ADC ratios (normalized to nontumorous tissues) [22], perform whole lesion measurement and/or generate histogram analysis including median and low percentile ADC values [23].

Entropy is a measure of image 'busyness/irregularity' and, as such, the observation of significantly lower mean TZ entropy values in patients with significant cancer would reflect increased overall signal homogeneity. Post-contrast T1 weighted image entropy was the second best univariate classifier, in keeping with previously observed homogenous enhancement features of TZ tumours [20]. However, the observation that the significant difference in $\mathrm{T} 1$ entropy between $\mathrm{TZ}$ containing significant tumours and non-significant/benign TZ persists even after the exclusion of radiologically visible tumours in the former group reflected more homogeneous enhancement throughout the whole TZ in these cases. The mechanism for this is unclear, though growing evidence implicates chronic inflammation as a contributor to prostate cancer development and progression [24] and present in benign prostate tissue associated with high-grade prostate cancer [25]. The finding of more homogenous enhancement in the 'non-malignant' parts of TZ harbouring significant tumours may reflect these aspects of prostate cancer ontogeny. ADC entropy was also significantly lower in single-slice TZ ROIs containing tumours and this relationship persisted following removal of cancer from the TZ ROI possibly due to the same/ similar mechanism. Additionally, at the time these data were acquired, as demonstrated in Table 2, the lowest b-value used for calculation of the ADC map was $0 \mathrm{sec} / \mathrm{mm} 2$, and it is possible the findings of ADC entropy may at least in part reflect a perfusion difference component. These findings may not have been appreciated in prior quantitative imaging studies, most of which concentrate on assessing the vascular properties of tumours themselves, and further confirmatory (histologically correlative) studies are required.

Several T2 weighted features aid visual identification of TZ tumours; including homogeneously reduced lesion signal intensity, ill-defined lesion margins and lenticular lesion shape [19]. However, on a whole TZ basis, the current study does not confirm that quantitatively reduced whole TZ entropy (increased homogeneity) on T2 weighted images is a particularly good discriminator. This may relate to the relatively small area of tumours compared with background non-tumorous $\mathrm{TZ}$ and/ or relate to the study size. Additionally, ADC and early postcontrast $\mathrm{T} 1$ images reflect processes occurring on a 'microscopic' level and can reflect field changes (for example, subtle inflammatory change) that may explain the significance of differences between benign $\mathrm{TZ}$ and that harbouring significant tumours. Whilst several studies have described homogenous reduced tumoral $\mathrm{T} 2$ signal compared to benign $\mathrm{TZ}$, the 
remaining (and predominant approximately $95 \%$ by area) 'normal' TZ glandular tissue is variably heterogeneous across patients and images are more reflective of macroscopic differences and subtle changes in $\mathrm{T} 2$ signal induced by background inflammation may be masked.

Skewness is a measure of histogram asymmetry; a zero value indicates a symmetrical distribution around the mean. Skewness was a relatively poor classifier of $\mathrm{TZ}$ cancer when applied to any image sequence. It has been speculated [23] that in prostate tumours densely packed with malignant cells, the resulting histograms from tumour ROIs are likely to be less skewed compared with tumours with more heterogeneous cellular density. However, we evaluated skewness for all pixels within the whole $\mathrm{TZ}$, and the proportion of tumour pixels may have been insufficient to influence skewness significantly particularly given such variation in cellular density is not captured by traditional histological grading [26].

To our knowledge, this study is distinct from other work as it assessed in vivo textural features derived from each mpMRI sequence for the entire TZ. The study was conducted as a proof of concept for the purpose of detecting significant TZ tumours. Future work will examine the translation of this concept over whole $\mathrm{TZ}$ volume to examine utility in augmenting visual radiologist assessment (e.g., added value of prompting readers to areas for examination which may contain significant cancer) and/or automated detection. Interpretation of images by radiologists is complex [27] and, although an assessment of texture is made, to date this visual textural assessment has not been characterised objectively. We believe that textural analysis of each multiparametric image is analogous to the manner by which radiologists visually localise $\mathrm{TZ}$ cancer on mpMRI [4]. Other workers [e.g., 28, 29] have examined first and second order (e.g., two-dimensional grey-level cooccurrence matrix) textural features from one or more mpMRI sequences from ROIs drawn around individual $\mathrm{TZ}$ tumours. Such approaches, while informative, have less clinical application, since they are more computationally intensive and require the radiologist to first identify areas of concern. Furthermore, where textural features of small lesions are evaluated at larger spatial scales, these features can be biased significantly by region boundary. In contrast, assessment of the entire $\mathrm{TZ}$ texture requires less development as a clinical diagnostic tool (i.e., only segmentation of the TZ from the PZ, which may be an automated/semi-automated procedure [30]) and minimizes boundary-related effects.

Our study has some limitations. All mpMRI images were acquired on a $1.5 \mathrm{~T}$ scanner and generalizability to $3 \mathrm{~T}$ platforms was not assessed. However, our 1.5 T mpMRI protocol was in keeping with recommendations from a European Consensus meeting [31]. We did not use endorectal coils, and note that the European Consensus Meeting failed to agree regarding their necessity even at $1.5 \mathrm{~T}$. We routinely perform DCE MRI using a higher spatial resolution and lower temporal resolution than some other centres as advocated by recent guidelines [3, 32]. Therefore, we expect the generalizability of post-contrast $\mathrm{T} 1$ weighted textural feature findings to be limited to centres that similarly maintain higher spatial resolution. We did not have access to radical prostatectomy specimens as a reference ground-truth. However, employment of a TPM based reference standard [33], which is a significant improvement over conventional TRUS biopsy [14], can help avoid the spectrum bias towards more severe cases that occurs with studies when prostatectomy specimens are mandated. We acknowledge that there could be some error when registering TPM histopathology to mpMRI. However, it should be noted that prostatectomy specimens themselves are not free from registration errors induced by processing (shrinkage, distortion, and tissue-loss) [34]. Lastly, we manually contoured the single-slice TZ ROIs though we do not expect this to unduly affect the results and envisage automated segmentation could be used as available.

Our results have immediate clinical relevance, and confirm textural features may inform CAD software to highlight images on which significant tumours could be present within the TZ. In developing this whole TZ approach using MRTA as an imaging biomarker, it is recognised that a series of studies would be required to further validate the findings as per various 'roadmap' approaches in contemporaneous guidelines (http://www.cancerresearchuk.org/sites/default/files/imaging biomarker_roadmap_for_cancer_studies.pdf). This approach could be adapted to several scenarios, subject to demonstration of efficacy, for example, by drawing attention to slice(s), which may require further examination for radiologists, probability of significant tumours being present across entire $\mathrm{TZ}$ and predictive information if there are relevant 'field' changes across the TZ. The United Kingdom NICE guidelines have adopted mpMRI for detection of prostate cancer in patients with a negative non-targeted TRUS biopsy (http://www.nice.org.uk/guidance/cg175 /chapter/recommendations). Our textural evaluation technique may have particular relevance for such patients who are more likely to have TZ tumours that are systematically undersampled by TRUS [34].

Acknowledgements The scientific guarantor of this publication is Shonit Punwani.

The authors of this manuscript declare relationships with the following companies:

BG- is a director of TexRAD Ltd (part of Feedback Plc, Cambridge, England) and Feedback Plc which provided the software and processing of images for analysis in this study. BG is not paid for board membership by TexRAD Ltd and Feedback Plc, is employed by TexRAD Ltd/ University College London, is not paid for patents (patent for TexRAD technique is transferred to TexRAD Ltd by the University of Sussex, United Kingdom) by TexRAD Ltd/University of Sussex and has not received any royalties; receives funds for travel for conferences and meetings if related to company work from TexRAD Ltd; holds shares in Feedback Plc. 
This study has received funding by: HSS and SP were supported by the KCL-UCL Comprehensive Cancer Imaging Centre funding [Cancer Research UK (CR-UK) \& Engineering and Physical Sciences Research Council (EPSRC)]. The majority of this work was undertaken at University College London Hospital and University College London, which receive a proportion of funding from the NIHR Biomedical Research Centre funding scheme [Department of Health UK].

One of the authors (Nikos Dikaios) has significant statistical expertise (in computational analysis procedures).

Institutional Review Board approval was obtained. Written informed consent was waived by the Institutional Review Board. No study subjects or cohorts have been previously reported.

Methodology: retrospective, diagnostic or prognostic study, performed at one institution.

Open Access This article is distributed under the terms of the Creative Commons Attribution 4.0 International License (http:// creativecommons.org/licenses/by/4.0/), which permits unrestricted use, distribution, and reproduction in any medium, provided you give appropriate credit to the original author(s) and the source, provide a link to the Creative Commons license, and indicate if changes were made.

\section{References}

1. Delongchamps NB, Rouanne M, Flam T, Beuvon F, Liberatore M, Zerbib M et al (2011) Multiparametric magnetic resonance imaging for the detection and localization of prostate cancer: combination of T2-weighted, dynamic contrast-enhanced and diffusion-weighted imaging. BJU Int 107:1411-1811

2. Langer DL, van der Kwast TH, Evans AJ, Trachtenberg J, Wilson BC, Haider MA (2009) Prostate cancer detection with multiparametric MRI: logistic regression analysis of quantitative T2, diffusion-weighted imaging, and dynamic contrast-enhanced MRI. J Magn Reson Imaging 30:327-334

3. Barentsz JO, Richenberg J, Clements R, Choyke P, Verma S, Villeirs G et al (2012) ESUR prostate MR guidelines 2012. Eur Radiol 22:746-757

4. Davnall F, Yip CS, Ljungqvist G, Selmi M, Ng F, Sanghera B et al (2012) Assessment of tumor heterogeneity: an emerging imaging tool for clinical practice? Insights Imaging 3:573-589

5. Ganeshan B, Miles KA (2013) Quantifying tumour heterogeneity with CT. Cancer Imaging 13:140-149

6. Eliat PA, Olivie D, Saikali S, Carsin B, Saint-Jalmes H, de Certaines JD (2012) Can dynamic contrast-enhanced magnetic resonance imaging combined with texture analysis differentiate malignant glioneuronal tumors from other glioblastoma? Neurol Res Int 2012, 195176

7. Parikh J, Selmi M, Charles-Edwards G, Glendenning J, Ganeshan $\mathrm{B}$, Verma $\mathrm{H}$ et al (2014) Changes in primary breast cancer heterogeneity may augment midtreatment MR imaging assessment of response to neoadjuvant chemotherapy. Radiology 272:100-112

8. De Cecco CN, Ganeshan B, Ciolina M, Rengo M, Meinel FG, Musio D et al (2015) Texture analysis as imaging biomarker of tumoral response to neoadjuvant chemoradiotherapy in rectal cancer patients studied with 3-T magnetic resonance. Invest Radiol 50: 239-245

9. Miles KA, Ganeshan B, Hayball MP (2013) CT texture analysis using the filtration-histogram method: what do the measurements mean? Cancer Imaging 13:400-406

10. Onik G, Barzell W (2008) Transperineal 3D mapping biopsy of the prostate: an essential tool in selecting patients for focal prostate cancer therapy. Urol Oncol 26:506-510
11. Taira AV, Merrick GS, Galbreath RW et al (2010) Performance of transperineal template-guided mapping biopsy in detecting prostate cancer in the initial and repeat biopsy setting. Prostate Cancer Prostatic Dis 13:71-77

12. Barzell WE, Melamed MR (2007) Appropriate patient selection in the focal treatment of prostate cancer: the role of transperineal 3 dimensional pathologic mapping of the prostate - a 4-year experience. Urology 70:27-35

13. Lecornet E, Ahmed HU, Hu Y, Moore CM, Nevoux P, Barratt D et al (2012) The accuracy of different biopsy strategies for the detection of clinically important prostate cancer: a computer simulation. J Urol 188:974-980

14. Ahmed HU, Hu Y, Carter T et al (2011) Characterizing clinically significant prostate cancer using template prostate mapping biopsy. J Urol 186:458-464

15. Stamey TA, Freiha FS, McNeal JE et al (1993) Localized prostate cancer. Relationship of tumor volume to clinical significance for treatment of prostate cancer. Cancer 71:933-938

16. Hanley JA, McNeil BJ (1982) The meaning and use of the area under a Receiver Operating Characteristic (ROC) curve. Radiology 143:29-36

17. Fukunaga K, Hummels DM (1989) Leave-one-out procedures for nonparametric error estimates. IEEE Trans Pattern Anal Mach Intell $11: 421-423$

18. Dikaios N, Alkalbani J, Sidhu HS, Fujiwara T, Abd-Alazeez M, Kirkham A et al (2015) Logistic regression model for diagnosis of TZ prostate cancer on multi-parametric MRI. Eur Radiol 25: 523-532

19. Akin O, Sala E, Moskowitz CS et al (2006) Transition zone prostate cancers: features, detection, localization, and staging at endorectal MR imaging. Radiology 239:784-792

20. Oto A, Kayhan A, Jiang Y et al (2010) Prostate cancer: differentiation of central gland cancer from benign prostatic hyperplasia by using diffusion-weighted and dynamic contrast-enhanced MR imaging. Radiology 257:715-723

21. Wibmer A, Hricak H, Gondo T, Matsumoto K, Veeraraghavan H, Fehr D et al (2015) Haralick texture analysis of prostate MRI: utility for differentiating non-cancerous prostate from prostate cancer and differentiating prostate cancers with different Gleason scores. Eur Radiol 25:2840-2850

22. Coffey N, Schieda N, Cron G, Gulavita P, Mai KT (2015) Flood TA (2014). Multi-parametric (mp) MRI of prostatic ductal adenocarcinoma. J Magn Reson Imaging 41:1639-1645

23. Donati OF, Mazaheri Y, Afaq A et al (2014) (2014) Prostate cancer aggressiveness: assessment with whole-lesion histogram analysis of the apparent diffusion coefficient. Radiology 271:143-152

24. Stark T, Livas L, Kyprianou N (2015) Inflammation in prostate cancer progression and therapeutic targeting. Transl Androl Urol 4:455-463

25. Gurel B, Lucia MS, Thompson IM Jr et al (2014) Chronic inflammation in benign prostate tissue is associated with high-grade prostate cancer in the placebo arm of the prostate cancer prevention trial. Cancer Epidemiol Biomarkers Prev 23:847-856

26. Langer DL, van der Kwast TH, Evans AJ et al (2008) Intermixed normal tissue within prostate cancer: effect on MR imaging measurements of apparent diffusion coefficient and T2 - sparse versus dense cancers. Radiology 249:900-908

27. Koontz NA, Gunderman RB (2008) Gestalt theory: implications for radiology education. AJR Am J Roentgenol 190:1156-1160

28. Vignati A, Mazzetti S, Giannini V, Russo F, Bollito E, Porpiglia F et al (2015) Texture features on T2-weighted magnetic resonance imaging: new potential biomarkers for prostate cancer aggressiveness. Phys Med Biol 60:2685-2701

29. Niaf E, Rouvière O, Mège-Lechevallier F, Bratan F, Lartizien C (2012) Computer-aided diagnosis of prostate cancer in the 
peripheral zone using multiparametric MRI. Phys Med Biol 57: 3833-3851

30. Garvey B, Türkbey B, Truong H, Bernardo M, Periaswamy S, Choyke PL (2014) Clinical value of prostate segmentation and volume determination on MRI in benign prostatic hyperplasia. Diagn Interv Radiol 20:229-233

31. Dickinson L, Ahmed HU, Allen C et al (2011) Magnetic resonance imaging for the detection, localisation, and characterisation of prostate cancer: recommendations from a European consensus meeting. Eur Urol 59:477-494
32. Kirkham AP, Haslam P, Keanie JY, McCafferty I, Padhani AR, Punwani S et al (2013) Prostate MRI: who, when, and how? Report from a UK consensus meeting. Clin Radiol 68: 1016-1023

33. Ahmed HU, Emberton M, Kepner G, Kepner J (2012) A biomedical engineering approach to mitigate the errors of prostate biopsy. Nat Rev Urol 9:227-23131

34. Xu S, Kruecker J, Turkbey B et al (2008) Real-time MRI-TRUS fusion for guidance of targeted prostate biopsies. Comput Aided Surg 13:255-264 